\title{
Teor e dinâmica do carbono no solo em sistemas de integração lavoura-pecuária
}

\author{
Júlio Cesar Salton(1), João Mielniczuk ${ }^{(2)}$, Cimélio Bayer ${ }^{(2)}$, Amoacy Carvalho Fabrício(1), \\ Manuel Cláudio Motta Macedo(3) e Dirceu Luiz Broch(4)
}

(1)Embrapa Agropecuária Oeste, BR 163, Km 253,6, CEP 79804-970 Dourados, MS. E-mail: salton@cpao.embrapa.br, amoacycf@yahoo.com.br (2)Universidade Federal do Rio Grande do Sul, Faculdade de Agronomia, Departamento de Solos, Caixa Postal 776, CEP 90001-970 Porto Alegre, RS. E-mail: mieln@ufrgs.br, bayer@ufrgs.br (3)Embrapa Gado de Corte, BR 262, Km 4, CEP 79002-970 Campo Grande, MS. E-mail: macedo@cnpgc.embrapa.br ${ }^{(4)}$ Fundação MS, Estrada da Usina Velha, Km 2, CEP $79150-000$ Maracaju, MS. E-mail: dirceu@fundacaoms.org.br

Resumo - O objetivo deste trabalho foi avaliar a capacidade de sistemas de manejo do solo alterarem os teores e a dinâmica do carbono no solo. Foram avaliados a vegetação natural e sistemas de manejo compostos de pastagens, lavouras anuais em preparo convencional e em plantio direto, e rotação de pastagem com lavoura, em experimentos de longa duração conduzidos em Dourados, Maracaju e Campo Grande, MS. Os experimentos foram dispostos em delineamento inteiramente casualizado, em Dourados e Maracaju, e em blocos ao acaso em Campo Grande. Foram determinados os teores e os estoques de $\mathrm{C}$ total no solo e em frações granulométricas da matéria orgânica do solo (MOS). A qualidade da MOS foi estimada pela relação entre o C nas frações lábil e não lábil, e expressa na forma de labilidade. A pastagem, permanente ou em rotação com lavouras, aumentou o C na fração particulada e a labilidade da MOS. As menores taxas de acúmulo e os menores estoques de $\mathrm{C}$ no solo foram observados nos sistemas apenas com lavouras anuais, enquanto os maiores valores foram registrados nos sistemas com pastagens.

Termos para indexação: Urochloa decumbens, fração lábil, pastagens, plantio direto, qualidade da matéria orgânica.

\section{Contents and dynamics of soil carbon in integrated crop-livestock systems}

\begin{abstract}
The objective of this work was to evaluate the ability of soil management systems to change the contents and dynamics of soil carbon. Natural vegetation and systems composed of perennial tropical pastures, annual crops under conventional tillage and no-tillage systems, and pasture rotation with annual crops were evaluated in long-term experiments carried out in Dourados, Maracaju and Campo Grande, Mato Grosso do Sul state, Brazil. The experiments were set in a completely randomized block design, in Dourados and Maracaju, and in a randomized complete block design in Campo Grande. Contents and stocks of total $\mathrm{C}$ in soil and in soil organic matter (SOM) fractions were measured. The quality of SOM was estimated by the ratio of C in labile and nonlabile fractions, and expressed in the form of lability. Permanent pasture or pasture in rotation with crops increased $\mathrm{C}$ in the particulate fraction and the SOM lability. Lower rates of accumulation and of stocks of soil $\mathrm{C}$ were observed in systems explored only with annual crops, while higher values were recorded in systems with pasture.
\end{abstract}

Index terms: Urochloa decumbens, labile fraction, pastures, no-tillage, organic matter quality.

\section{Introdução}

A manutenção dos resíduos vegetais sobre a superfície do solo e a redução em seu revolvimento são apontados como meios para aumentar o armazenamento de carbono no solo. Sistemas de preparo convencional com revolvimento do solo por gradagem apresentam decréscimo expressivo nos estoques de matéria orgânica do solo (MOS), em comparação ao plantio direto (PD) (Leite et al., 2010; Teixeira et al., 2010). A magnitude desses efeitos depende do clima, do tipo de solo e dos sistemas de manejo utilizados. Em regiões tropicais, as taxas de perda da matéria orgânica (MO) são até cinco vezes maiores do que em regiões temperadas (Sanchez \& Logan, 1992).

Além da ausência de revolvimento do solo, a adoção de sistemas de rotação que incluam culturas com grande formação de fitomassa (aérea e radicular) de elevada relação C:N é fundamental para preservação da MOS (Bayer \& Mielniczuk, 1999; Bayer et al., 2000). As culturas escolhidas para exploração do solo também podem contribuir para o aumento do estoque de $\mathrm{C}$ no solo, como relatado por Diekow (2003), que verificou 45,4 $\mathrm{Mg} \mathrm{ha}^{-1}$ de C na camada 0 a 17,5 $\mathrm{cm}$ de um Argissolo

Pesq. agropec. bras., Brasília, v.46, n.10, p.1349-1356, out. 2011 
do Rio Grande do Sul, no sistema guandu/milho, em comparação a 29,2 $\mathrm{Mg} \mathrm{ha}^{-1}$ de C no sistema aveia/milho, ambos cultivados em PD durante 18 anos, com estoque inicial de $32,5 \mathrm{Mg} \mathrm{ha}^{-1}$. Em sistemas de integração lavoura-pecuária, a forma de manejo das pastagens e a lotação animal, por resultarem em diferentes quantidades de massa de forragem e nutrientes reciclados, podem aportar diferentes quantidades de $\mathrm{C}$ ao solo (Nicoloso et al., 2006; Lopes et al., 2008, Souza et al., 2008; Carvalho et al., 2010).

Sistemas de produção que resultam no aumento dos teores daMOS têm sido apontados como mais sustentáveis, uma vez que a matéria orgânica relaciona-se a múltiplos aspectos do ambiente e da qualidade do solo, o que a torna um dos principais responsáveis pela sustentabilidade dos sistemas agropecuários (Mielniczuk et al., 2003). Para que a utilização da MOS como indicadora da qualidade do solo seja útil na identificação das formas de manejo mais sustentáveis, é preciso conhecer com detalhe os mecanismos de aporte de carbono ao solo e a sua dinâmica. Postula-se, como hipótese geral, que a introdução de pastagens de forma alternada com lavouras de soja em plantio direto resulta em maiores estoques de carbono no solo do que sistemas contínuos ou monocultivos.

O objetivo deste trabalho foi avaliar a capacidade de sistemas de manejo do solo alterarem os teores e a dinâmica do carbono no solo.

\section{Material e Métodos}

As avaliações foram realizadas em experimentos de campo, com 9 e 11 anos de duração, em sistemas de manejo com histórico conhecido, em Dourados, Campo Grande e Maracaju, MS. As principais características dos experimentos estão sistematizadas e apresentadas na Tabela 1; maiores detalhes podem ser encontrados em Salton (2005). O delineamento experimental utilizado em Dourados e Maracaju foi o inteiramente casualizado e, em Campo Grande, o de blocos ao acaso.

Os sistemas de manejo avaliados foram: L-PC, lavoura em preparo convencional do solo, com uso de grades de discos (pesada + niveladora), com soja no verão e aveia no inverno, em Dourados, e apenas soja em Campo Grande; L-PD, lavoura em plantio direto, com a sequência soja/ aveia em Maracaju, nabo/milho/aveia/soja/trigo/soja em Dourados e milheto/soja até 2000, seguidos por sorgo/ soja, em Campo Grande; S2P2d, lavoura de soja em rotação com pastagem de Urochloa decumbens (syn.
Brachiaria decumbens), com ciclos de dois anos de lavoura e dois anos de pastagem, com implantação da soja sobre a pastagem e da pastagem após a soja, em plantio direto - entre os dois cultivos de soja (outono/ inverno), foi utilizada aveia para cobertura do solo e pastejo, e feita adubação da soja com $300 \mathrm{~kg} \mathrm{ha}^{-1}$ da fórmula 00-20-20; S1P3, lavoura de soja em plantio direto em rotação com pastagem (U. brizantha), com ciclos de um ano de lavoura e três anos de pastagem; S4P4, lavoura de soja em plantio direto em rotação com pastagem de Panicum maximum cv. Tanzânia, com ciclos de quatro anos de lavoura e quatro anos de pastagem adubada anualmente (200 $\mathrm{kg} \mathrm{ha}^{-1}$ da fórmula 00-20-20 + $100 \mathrm{~kg} \mathrm{ha}^{-1} \mathrm{de}$ $\mathrm{N})$ - entre os cultivos de soja (outono/inverno), foi utilizado milheto ou sorgo para cobertura do solo e pastejo; PPd, pastagem permanente de $U$. decumbens; $\mathrm{PPb}$, pastagem permanente de $U$. brizantha; $\mathrm{PP}+\mathrm{L}$, pastagem permanente com $U$. decumbens implantada em dezembro de 1993, consorciada com leguminosas (Stylosanthes guianensis, S. macrocarpa, S. macrocephala e Calopogonium spp.), com adubação de manutenção a cada dois anos (400 $\mathrm{kg} \mathrm{ha}^{-1}$ de 0-20-20), e submetida à pastejo contínuo; e VN, vegetação natural, conforme Tabela 1.

Em todos os locais, foram utilizados bovinos de corte submetidos aos sistemas de manejo descritos na Tabela 1 . Próximo à área de cada experimento, foram amostradas áreas não perturbadas, com vegetação original, utilizada como referência.

Entre 12 e 17 de abril de 2004, coletaram-se amostras para determinação do teor de carbono orgânico nas camadas $0-2,5,2,5-5,5-10$ e 10-20 cm, além de amostras indeformadas, com uso de anel metálico, para determinação da densidade do solo. Todas as amostragens foram efetuadas com três repetições, exceto as de densidade do solo, que foram seis.

O carbono orgânico do solo (COT) foi expresso quanto ao teor $\left(\mathrm{g} \mathrm{kg}^{-1}\right)$ e ao estoque $\left(\mathrm{Mg} \mathrm{ha}^{-1}\right)$ nas diferentes camadas do solo, este último calculado de acordo com os valores de densidade do solo: estoque $=$ teor de $\mathrm{C}(\%) \mathrm{x}$ densidade do solo $\left(\mathrm{g} \mathrm{cm}^{-3}\right)$ $\mathrm{x}$ espessura da camada $(\mathrm{cm})$. A determinação dos teores de carbono foi realizada por combustão seca, e a quantificação do $\mathrm{CO}_{2}$ liberado, em sensor de infravermelho do analisador Shimadzu, modelo TOC-VCPN com SSM-5000A (Shimadzu do Brasil, São Paulo, SP), com uso de amostras 
de aproximadamente $0,5 \mathrm{~g}$ maceradas em almofariz de ágata até passar em peneira com abertura de $0,25 \mathrm{~mm}$.

$\mathrm{O}$ teor de $\mathrm{C}$ nas frações da MOS foi obtido a partir do fracionamento físico granulométrico, de acordo com o método descrito por Cambardella \& Elliott (1993); este protocolo foi empregado na análise de amostras das três localidades. O fracionamento da MOS consistiu na extração de uma subamostra, que foi seca ao ar e moída com rolo até passar em peneira com abertura de $2 \mathrm{~mm}$; foram excluídos materiais vegetais grosseiros, como pedaços de hastes, talos e parte superior de raízes (touceiras). A subamostra, com $20 \mathrm{~g}$ de solo, foi acondicionada em frascos tipo "snap-cap" e agitada horizontalmente durante 15 horas em solução de $\left(\mathrm{NaPO}_{3}\right)_{\mathrm{n}}(0,5 \%)$. A eficiência da dispersão do solo foi avaliada visualmente em lupa. Em seguida, o conteúdo dos frascos foi peneirado, com auxílio de jatos d'água, em peneira com malha com abertura de $0,053 \mathrm{~mm}$, limite superior do diâmetro da partícula de silte, pela escala americana. A fração da amostra retida na peneira foi seca em estufa a $50^{\circ} \mathrm{C}$. Então, foi determinada a massa da amostra, que foi moída em almofariz para análise do teor de carbono; a partir deste procedimento, obteve-se o teor de carbono (\%) na fração particulada da matéria orgânica (C-MOP). O carbono da fração não retida na peneira, constituída de material de tamanho correspondente a silte e argila, foi considerado associado aos minerais do solo e, portanto, não lábil. $\mathrm{O}$ teor de carbono nesta fração foi obtido pela subtração do valor de C-MOP do COT, denominado C-MOM.

Os dados foram submetidos à análise de variância, e quando diferenças significativas foram observadas, as médias foram comparadas pelo teste DMS, a 5\% de probabilidade. O tratamento dos dados foi realizado com os programas Excel e Sigmaplot.

\section{Resultados e Discussão}

$\mathrm{O}$ teor de $\mathrm{C}$ foi maior nas camadas superficiais do solo e decresceu com a profundidade, em todos os tratamentos das três localidades, exceto nos sistemas L-PC e PPd em Campo Grande, nos quais a concentração de $\mathrm{C}$ foi maior na profundidade de 5 a $10 \mathrm{~cm}$ (Figura 1). O sistema L-PD, com aporte de resíduos semelhante aos demais, apresentou maior concentração de $\mathrm{C}$ na camada superficial, em virtude

Tabela 1. Caracterização das áreas experimentais e do solo em três localidades, em Mato Grosso do Sul, a partir de valores médios de amostras compostas retiradas da camada $0-20 \mathrm{~cm}$.

\begin{tabular}{|c|c|c|c|}
\hline Característica & Dourados & Maracaju & Campo Grande \\
\hline Localização & Embrapa Agropecuária Oeste & Fundação MS & Embrapa Gado de Corte \\
\hline Coordenadas & $22^{\circ} 14^{\prime} \mathrm{S} 54^{\circ} 49^{\prime} \mathrm{W}$ & $21^{\circ} 36^{\prime} \mathrm{S} 55^{\circ} 10^{\prime} \mathrm{W}$ & $20^{\circ} 26^{\prime} \mathrm{S} 54^{\circ} 43^{\prime} \mathrm{W}$ \\
\hline Altitude (m) & 430 & 390 & 530 \\
\hline Clima & $\begin{array}{l}\text { Cwa - mesotérmico úmido, } \\
\text { verões quentes e invernos secos }\end{array}$ & $\begin{array}{l}\text { Cwa - mesotérmico úmido, } \\
\text { verões quentes e invernos secos }\end{array}$ & $\begin{array}{l}\text { Aw - tropical úmido, verões } \\
\text { chuvosos e invernos secos }\end{array}$ \\
\hline Vegetação natural & Campo ralo & Campo ralo & Cerrado sensu stricto \\
\hline Uso anterior ao experimento & $\begin{array}{c}+/-30 \text { anos de lavouras de grãos } \\
\text { com preparo convencional }\end{array}$ & $\begin{array}{l}\text { Sem registro de utilização } \\
\text { agrícola ou pastoril }\end{array}$ & $\begin{array}{c}\text { Pastagem degradada estabelecida } \\
\text { em } 1973 \text { sobre área bruta }\end{array}$ \\
\hline Início do experimento & 1995 & 1993 & 1993 \\
\hline Área (ha) & 28 & 1,2 & 28,2 \\
\hline Tipo de solo & LVdf & LVdf & LV \\
\hline $\mathrm{pH}$ em $\mathrm{H}_{2} \mathrm{O}$ & 5,33 & 5,70 & 5,14 \\
\hline $\mathrm{Al}\left(\mathrm{cmol}_{\mathrm{c}} \mathrm{dm}^{-3}\right)$ & 0,76 & 0,0 & 0,95 \\
\hline $\mathrm{Ca}\left(\mathrm{cmol}_{\mathrm{c}} \mathrm{dm}^{-3}\right)$ & 4,00 & 7,50 & 0,55 \\
\hline $\operatorname{Mg}\left(\mathrm{cmol}_{\mathrm{c}} \mathrm{dm}^{-3}\right)$ & 1,90 & 1,70 & 0,26 \\
\hline P-Mehlich $\left(\mathrm{mg} \mathrm{dm}^{-3}\right)$ & 11,80 & 8,10 & 1,55 \\
\hline $\mathrm{K}\left(\mathrm{cmol}_{\mathrm{c}} \mathrm{dm}^{-3}\right)$ & 0,37 & 0,27 & 0,11 \\
\hline Argila, silte e areia $\left(\mathrm{g} \mathrm{kg}^{-1}\right)$ & $640,210,150$ & $530,200,270$ & $360,130,510$ \\
\hline Densidade de partícula $\left(\mathrm{Mg} \mathrm{m}^{-3}\right)$ & 2,92 & 2,88 & 2,67 \\
\hline Fe total $(\%)$ & 11,43 & 12,48 & 12,89 \\
\hline $\mathrm{Hm} /(\mathrm{Hm}+\mathrm{Gt})$ & 0,97 & 0,92 & 0,71 \\
\hline $\mathrm{Gb} /(\mathrm{Gb}+\mathrm{Ct})$ & 0,04 & 0,22 & 0,47 \\
\hline Critério de manejo da pastagem & $\begin{array}{l}\text { Manutenção da oferta de } \\
\text { forragem em torno de } 7 \%\end{array}$ & $\begin{array}{c}\text { Pastejo esporádico para manutenção } \\
\text { da pastagem com cerca de } 40 \mathrm{~cm}\end{array}$ & $\begin{array}{c}\text { Manutenção da massa de matéria } \\
\text { seca total em } 3 \mathrm{Mg} \mathrm{ha}^{-1}\end{array}$ \\
\hline
\end{tabular}

LVdf, Latossolo Vermelho distroférico; LV, Latossolo Vermelho; Hm, hematita; Gt, goethita; Gb, gibbsita; Ct, caulinita. 


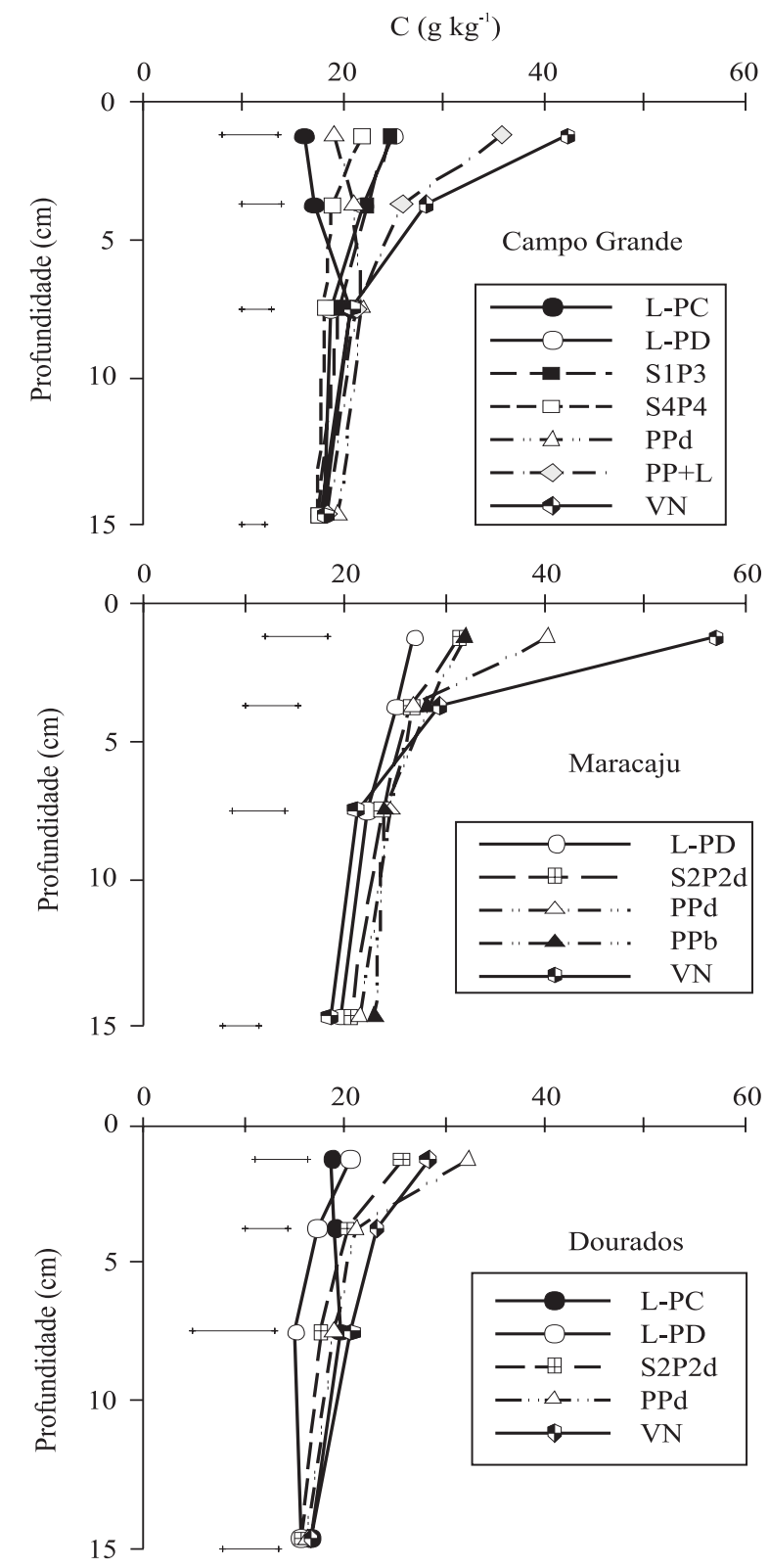

Figura 1. Teor de carbono em camadas de Latossolo Vermelho distroférico (LVdf), em Dourados, MS, submetido aos sistemas de manejo durante nove anos, e de LVdf em Maracaju, MS, e Latossolo Vermelho em Campo Grande, MS, submetidos a sistemas de manejo durante 11 anos. As barras indicam a DMS, a 5\% de probabilidade. L-PC, lavouras em sistema convencional; L-PD, lavouras em plantio direto; S2P2d, rotação de soja por dois anos com pastagem (Urochloa decumbens) por dois anos; PPd, pastagem permanente (U. decumbens); $\mathrm{PPb}$, pastagem permanente (U. brizantha); S1P3, rotação de soja por um ano com pastagem (U. brizantha) por três anos; $\mathrm{S} 4 \mathrm{P} 4$, rotação de soja por quatro anos com pastagem (Panicum maximum) por quatro anos; $\mathrm{PP}+\mathrm{L}$, pastagem permanente $(U$. decumbens) consorciada com leguminosas; $\mathrm{VN}$, vegetação natural. do acúmulo de resíduos sobre o solo, o que também foi verificado em outros experimentos de longa duração na região Sul do Brasil (Boddey et al., 2010).

No sistema com PPd em Campo Grande, o teor de $\mathrm{C}$ na superfície foi menor do que o das demais profundidades, o que pode estar associado ao manejo da pastagem, que não aportou $\mathrm{C}$ ao solo na quantidade suficiente para mantê-lo em equilíbrio. $\mathrm{O}$ acúmulo de $\mathrm{C}$ no solo pela pastagem depende da produtividade das plantas, como verificado por Lilienfein et al. (2003) que, ao comparar pastagens de $U$. decumbens, em condição degradada e produtiva, encontraram teores de $\mathrm{C}$ no solo $(0-15 \mathrm{~cm})$ de 22 e $27 \mathrm{~g} \mathrm{~kg}^{-1}$, respectivamente.

Em todos os sistemas de produção, foram observados teores de $\mathrm{C}$ semelhantes na camada mais profunda (Figura 1). No entanto, na camada $0-2,5 \mathrm{~cm}$ foram observadas diferenças significativas entre os sistemas, em todos os locais. Sistemas com pastagens, em comparação aos exclusivamente com lavouras (L-PD e L-PC), apresentaram maiores teores de $\mathrm{C}$, o que deve estar associado ao elevado aporte de material vegetal comumente proporcionado pelas pastagens. A utilização de diferentes culturas, com diferentes aportes de $\mathrm{C}$, resulta em diferentes estoques de $\mathrm{C}$ no solo, conforme relato de Diekow (2005). As pastagens apresentam a maior parte do $\mathrm{C}$ armazenado abaixo da superfície. Fujisaka et al. (1998) e Lal (2002), entre outros autores, atribuem ao sistema radicular de pastagens a grande capacidade de acumular $\mathrm{C}$ no solo.

No sistema $\mathrm{VN}$, os valores de estoque de $\mathrm{C}$ na camada $0-20 \mathrm{~cm}$ foram de aproximadamente 44,5, 68,7 e 54,0 $\mathrm{Mg} \mathrm{ha}^{-1}$ em Dourados, Maracaju e Campo Grande, respectivamente. Com a utilização agrícola dos solos, houve redução desses valores para 41,9, 51,7 e $51,3 \mathrm{Mg} \mathrm{ha}^{-1}$, respectivamente. Após a implantação dos experimentos, os estoques de $\mathrm{C}$ no solo foram alterados, com acréscimos nos sistemas com pastagens, que ultrapassaram os valores iniciais (VN) em Dourados e Campo Grande (Tabela 2). As taxas anuais de retenção de C na camada $0-20 \mathrm{~cm}$ variaram de $1,12 \mathrm{Mg} \mathrm{ha}^{-1}$ por ano, no sistema $\mathrm{PP}+\mathrm{L}$ em Campo Grande, a taxas praticamente nulas, nos sistemas apenas com lavouras (Tabela 2). Maiores taxas de acúmulo de $\mathrm{C}$ no solo estão associadas a maior aporte de material orgânico ao solo pelas culturas (Lovato et al., 2004), o que foi confirmado pelos fato de as maiores taxas coincidirem com a presença de pastagens. 
Atualmente, além da eficiência agronômica, os sistemas de produção devem proporcionar ganhos ambientais, como menor emissão de gases causadores de efeito estufa e sequestro de $\mathrm{C}$ atmosférico. Assim, o impacto proporcionado pela utilização da rotação de lavoura com pastagem, como o sistema S2P2, em substituição ao sistema L-PD predominante na região de Dourados, poderia causar retenção de $\mathrm{C}$ ao solo a uma taxa de 0,40 e $0,80 \mathrm{Mg} \mathrm{ha}^{-1}$ por ano, ao considerar-se os estoques na camada $0-20 \mathrm{~cm}$. Este valor é similar aos verificados por Bayer et al. (2004), ao comparar lavouras de grãos em PD e PC em Costa Rica, MS, em Latossolo com teor de argila semelhante ao do presente trabalho $\left(650 \mathrm{~g} \mathrm{~kg}^{-1}\right)$, e por Corazza et al. (1999) e Oliveira et al. (2004), que

Tabela 2. Estoques de carbono orgânico total (COT) na camada $0-20 \mathrm{~cm}$ do solo e taxas médias anuais $\left(\mathrm{Mg} \mathrm{ha}^{-1}\right.$ por ano) de retenção de carbono (C) no solo para diferentes sistemas de manejo, em três localidades do Estado do Mato Grosso do Sul.

\begin{tabular}{|c|c|c|c|c|}
\hline \multirow{2}{*}{$\begin{array}{l}\text { Sistemas } \\
\text { de manejo }\end{array}$} & \multicolumn{2}{|c|}{ Estoque de COT $\left(\mathrm{Mg} \mathrm{ha}^{-1}\right)$} & \multicolumn{2}{|c|}{ Taxa de retenção de C } \\
\hline & Início experimento & 2004 & Absoluta & Relativa $^{(1)}$ \\
\hline & \multicolumn{4}{|c|}{ Dourados } \\
\hline L-PC & 41,92 & 44,10 & $-0,0019^{(4)}$ & 0 \\
\hline L-PD & 41,92 & 42,60 & $-0,1687$ & $-0,1667$ \\
\hline S2P2d & 41,92 & 48,02 & 0,4400 & 0,4356 \\
\hline PPd & 41,92 & 50,11 & 0,9089 & 0,6678 \\
\hline \multirow[t]{2}{*}{$\mathrm{VN}$} & - & 44,49 & - & - \\
\hline & \multicolumn{4}{|c|}{ Maracaju } \\
\hline L-PD & $51,68^{(2)}$ & 56,60 & $0,4473^{(5)}$ & 0 \\
\hline $\mathrm{S} 2 \mathrm{P} 2 \mathrm{~d}$ & 51,68 & 61,39 & 0,8836 & 0,4354 \\
\hline PPd & 51,68 & 65,80 & 1,2836 & 0,8364 \\
\hline $\mathrm{PPb}$ & 51,68 & 66,97 & 1,3927 & 0,9427 \\
\hline \multirow[t]{2}{*}{$\mathrm{VN}$} & - & 68,66 & - & - \\
\hline & \multicolumn{4}{|c|}{ Campo Grande } \\
\hline L-PC & $51,30^{(3)}$ & 46,30 & $-0,4545^{(5)}$ & 0 \\
\hline L-PD & 51,30 & 47,40 & $-0,3545$ & 0,1000 \\
\hline S4P4 & 51,30 & 47,90 & $-0,3091$ & 0,1455 \\
\hline S1P3 & 51,30 & 50,50 & $-0,0727$ & 0,3818 \\
\hline PPd & 51,30 & 53,50 & 0,2000 & 0,6545 \\
\hline $\mathrm{PP}+\mathrm{L}$ & 51,30 & 58,60 & 0,6636 & 1,1182 \\
\hline $\mathrm{VN}$ & - & 54,00 & - & - \\
\hline
\end{tabular}

${ }^{(1)} \mathrm{L}-\mathrm{PC}$, lavouras em preparo convencional; L-PD, lavouras em plantio direto; $\mathrm{S} 2 \mathrm{P} 2 \mathrm{~d}$, rotação de soja por dois anos com pastagem (Urochloa decumbens) por dois anos; S4P4, rotação de soja por quatro anos com pastagem (Panicum maximum) por quatro anos; S1P3, rotação de soja por um ano com pastagem $(U$. brizantha) por três anos; PPd, pastagem permanente (U. decumbens); VN, vegetação natural. (1)Relativa à L-PC (Dourados e Campo Grande) ou L-PD (Maracaju) ${ }^{(2)}$ Valor obtido a partir de relatórios do experimento (Fundação MS/Embrapa). ${ }^{(3)}$ Valor estimado a partir de Macedo et al. (2001). ${ }^{(4)}$ Corresponde ao coeficiente angular da equação de ajuste linear para os dados do local (1996, 1998, 2001 e 2004). ${ }^{(5)}$ Valor obtido a partir da expressão y $=($ estoque 2004 - estoque inicial $) / 11$ anos. relataram taxas de 0,63 e $0,52 \mathrm{Mg} \mathrm{ha}^{-1}$, respectivamente, para Latossolo do DF com $410 \mathrm{~g} \mathrm{~kg}^{-1}$ de argila.

Na maioria das situações experimentais, o PD apresenta maior taxa de acúmulo de $\mathrm{C}$ em comparação ao PC. No entanto, Roscoe \& Buurman (2003) encontraram taxa negativa $\left(0,03 \mathrm{Mg} \mathrm{ha}^{-1}\right.$ por ano) ou praticamente nula no PD (milho, feijão), em comparação ao PC em Latossolo com $800 \mathrm{~g} \mathrm{~kg}^{-1}$ de argila, em Minas Gerais. Neste caso, o efeito da textura do solo parece sobrepujar o efeito da operação de preparo do solo, o que está de acordo com os resultados verificados em Dourados, em que as taxas de acúmulo foram praticamente nulas. No experimento de Campo Grande, implantado em solo com textura mais arenosa que os demais, os efeitos dos sistemas de manejo na MOS foram ampliados.

A maior quantidade de $\mathrm{C}$ na fração particulada da MOS (C-MOP) foi verificada na camada superficial, em todos os sistemas de manejo. A exceção foi o sistema L-PC, no qual o maior valor foi encontrado na camada $5-10 \mathrm{~cm}$, provavelmente em decorrência da deposição de material pelas gradagens durante o preparo do solo (Figura 2). Também foi verificada contribuição da pastagem na camada 0-2,5 cm; o sistema PP apresentou estoque de 2,9 $\mathrm{Mg} \mathrm{ha}^{-1}$ de C-MOP, enquanto o L-PC apresentou cerca de cinco vezes menos no experimento em Dourados, valor semelhante ao encontrado nos demais locais (Figura 2).

É desejável que o solo apresente quantidade adequada de C-MOP, pois, assim, garante-se o fluxo de $\mathrm{C}$ para o solo e a manutenção da atividade biológica. Caso o solo não disponha de matéria orgânica lábil em quantidade suficiente para suprir suas necessidades, os processos de oxidação da MOS resultarão em redução do estoque de $\mathrm{C}$, o que dá início ao processo de perda de qualidade e degradação do solo (Causarano et al., 2008). Ao apresentar 6,7 Mg ha ${ }^{-1}$ de C-MOP, o sistema PP foi significativamente superior aos sistemas L-PC e L-PD, com 3,6 e 4,1 $\mathrm{Mg} \mathrm{ha}^{-1}$ de C-MOP, respectivamente (Figura 2). Franzluebbers \& Stuedemann (2002) avaliaram a distribuição da MOS nas frações particulada e não particulada, em perfis do solo nos EUA, e encontraram valores maiores em pastagens e áreas sob pastejo do que em lavouras em preparo conservacionista e áreas para fenação. Isso demonstra a importância do material senescente e dos dejetos animais para o aumento dos teores de $\mathrm{C}$ no material particulado na pastagem, especialmente na camada superficial.

A maior sensibilidade da fração particulada da MOS, em comparação à fração associada aos minerais, em exibir os efeitos dos sistemas de manejo sobre a dinâmica 

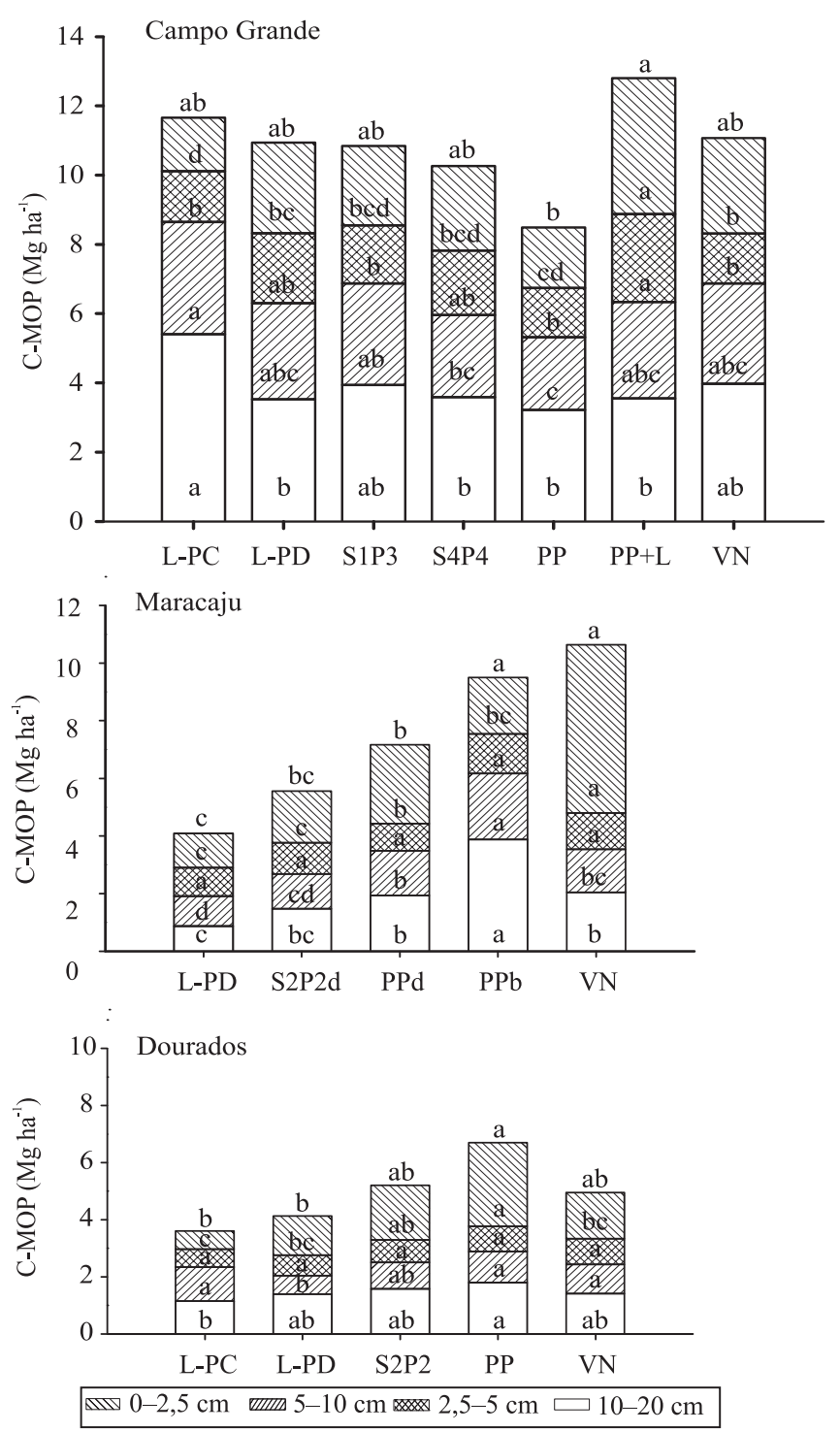

Figura 2. Estoques de carbono na matéria orgânica particulada (C-MOP) em camadas de Latossolo Vermelho distroférico (LVdf) em Dourados, MS, submetido a sistemas de manejo durante nove anos, e de LVdf em Maracaju, MS, e LV em Campo Grande, MS, submetidos a sistemas de manejo durante 11 anos. Letras iguais, para a profundidade no interior das barras e para a camada $0-20 \mathrm{~cm}$ no topo das barras, indicam semelhança pelo teste DMS, a 5\% de probabilidade. L-PC, lavouras em sistema convencional; L-PD, lavouras em plantio direto; S2P2d, rotação de soja por dois anos com pastagem (Urochloa decumbens) por dois anos; PPd, pastagem permanente ( $U$. decumbens); $\mathrm{PPb}$, pastagem permanente (U. brizantha); S1P3, rotação de soja por um ano com pastagem (U. brizantha) por três anos; S4P4, rotação de soja por quatro anos com pastagem (Panicum maximum) por quatro anos; $\mathrm{PP}+\mathrm{L}$, pastagem permanente ( $U$. decumbens) consorciada com leguminosas; $\mathrm{VN}$, vegetação natural. do carbono no solo pode ser observada nos estoques de C nas frações da MOS. Nos sistemas de manejo com culturas anuais, o rendimento das culturas, as quantidades de resíduos e as condições climáticas parecem ser determinantes das variações no estoque de C-MOP. Bayer et al.(2002) afirmam que a fração particulada da MOS é mais sensível às práticas de manejo do solo, pois alterase conforme as variações no aporte de material vegetal e nas taxas de decomposição promovidas por práticas de preparo do solo.

Os sistemas de manejo, ao aportarem diferentes quantidades de $\mathrm{C}$ ao solo, alteram a labilidade da MOS, ou seja, a proporção de MOS lábil em relação à não lábil $(\mathrm{Lab}=\mathrm{C}-\mathrm{MOP} / \mathrm{C}-\mathrm{MOM})$. No geral, os maiores valores foram verificados na camada superficial $(0-2,5 \mathrm{~cm})$ em virtude da grande quantidade de resíduos vegetais aportados na superfície do solo (Figura 3). Nesta camada, predominaram valores de labilidade entre 0,2 e 0,3 , para Dourados e Maracaju, enquanto em Campo Grande este valor foi de aproximadamente 0,4. Nas demais profundidades, em todos os locais estudados, a labilidade da MOS reduziu-se e atingiu valores inferiores a 0,1 , exceto no sistema L-PC, em que a labilidade da MOS nestas posições do perfil do solo é resultante da incorporação de resíduos vegetais (MOP) pelas gradagens. Vieira (2007) encontrou valores de labilidade da MOS entre 0,105 e 0,215 em sistemas com gramíneas sem adubação nitrogenada e em sucessão guandu/milho, respectivamente, similares aos de 0,10 a 0,15 obtidos por Souza et al. (2008) em sistema de integração lavoura-pecuária. O sistema $\mathrm{VN}$, em Maracaju, apresentou valor discrepante dos demais $(0,43)$ na camada de 0 a $2,5 \mathrm{~cm}$; os outros sistemas apresentaram valores entre 0,16 (L-PD) e 0,27 (PPb). O sistema $\mathrm{PPb}$ apresentou maior labilidade, o que indica ter ocorrido aporte de material particulado nas camadas mais profundas, provavelmente em decorrência do sistema radicular de $U$. brizantha. No experimento de Campo Grande, foi observado valor muito superior aos demais sistemas para L-PD $(0,72)$, o que pode ter sido consequência da amostragem do solo logo após a colheita da soja, e da quantidade de material depositado na camada superficial durante os 11 anos de condução do experimento. Os sistemas PP e L-PC distinguiram-se dos demais, nas camadas 5-10 e 10-20 cm, respectivamente. O sistema PP apresentou os menores valores de labilidade em todo o perfil, principalmente na camada $5-10 \mathrm{~cm}$, o que pode estar associado a um manejo inadequado da pastagem, 

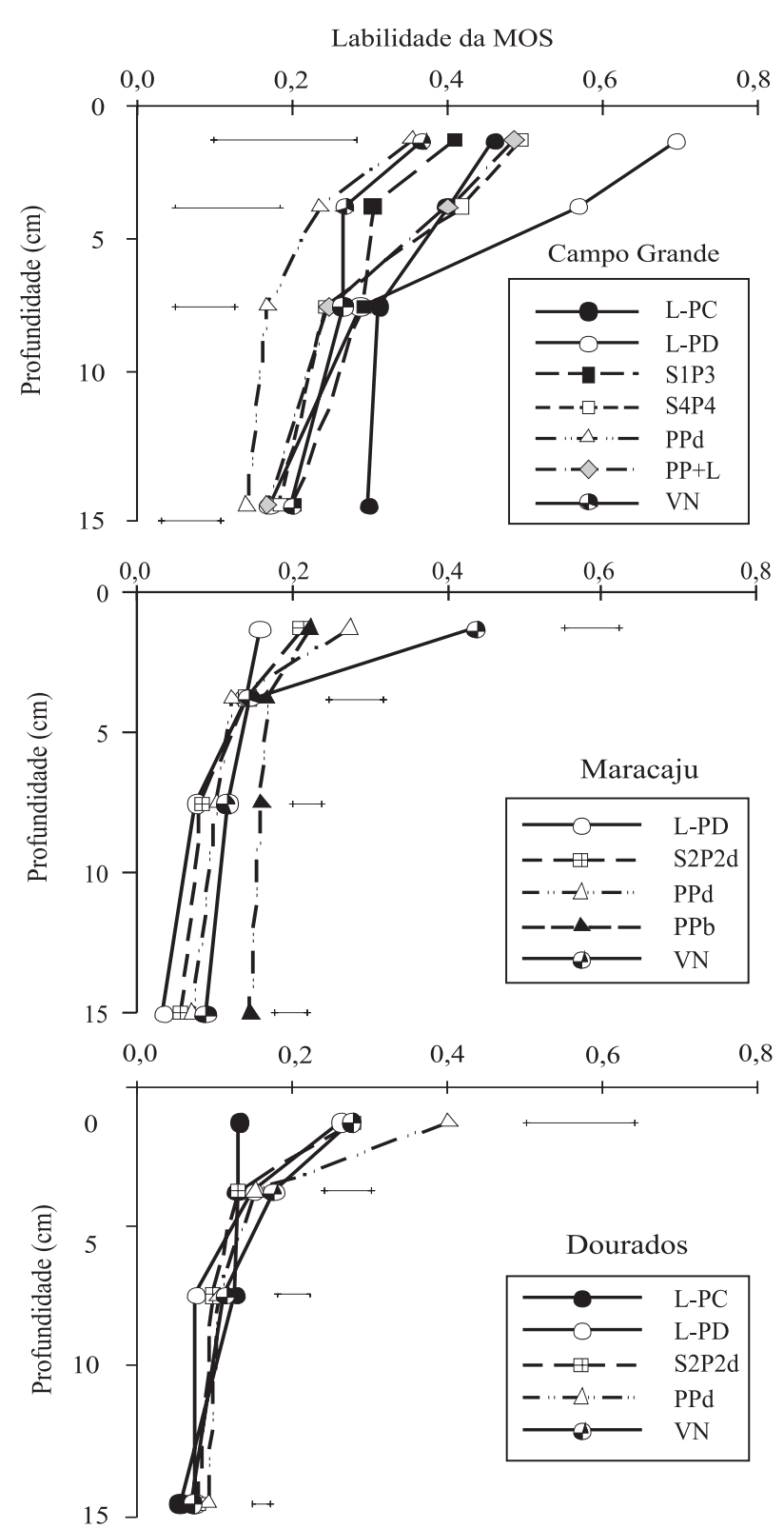

Figura 3. Labilidade da matéria orgânica do solo (MOS) de um Latossolo Vermelho distroférico (LVdf) em Dourados, MS, submetido a sistemas de manejo durante nove anos, e de LVdf em Maracaju, MS, e Latossolo Vermelho em Campo Grande, MS, submetidos a sistemas de manejo durante 11 anos. As barras indicam a DMS, a 5\% de probabilidade. L-PC, lavouras em sistema convencional; L-PD, lavouras em plantio direto; S2P2d, rotação de soja por dois anos com pastagem (Urochloa decumbens) por dois anos; PPd, pastagem permanente ( $U$. decumbens); $\mathrm{PPb}$, pastagem permanente (U. brizantha); $\mathrm{S} 1 \mathrm{P} 3$, rotação de soja por um ano com pastagem (U. brizantha) por três anos; S4P4, rotação de soja por quatro anos com pastagem (Panicum maximum) por quatro anos; $\mathrm{PP}+\mathrm{L}$, pastagem permanente $(U$. decumbens) consorciada com leguminosas; $\mathrm{VN}$, vegetação natural. que teria resultado em redução no aporte de material particulado. No sistema L-PC, ocorreu o inverso, com valores de labilidade significativamente superiores aos dos demais sistemas na camada $10-20 \mathrm{~cm}$, o que pode ser justificado pela incorporação dos resíduos vegetais pelo preparo do solo, associado ao severo deficit hídrico nos meses anteriores à amostragem, o que interferiu na taxa de decomposição do material vegetal.

\section{Conclusões}

1. As maiores taxas de acúmulo e os maiores estoques de carbono no solo ocorrem nos sistemas com pastagem permanente, enquanto os menores valores ocorrem nos sistemas com lavouras e os valores intermediários nos sistemas com integração lavoura-pastagem.

2. Os sistemas com pastagens apresentam os maiores estoques de carbono na fração particulada da matéria orgânica do solo.

3. A maior labilidade da matéria orgânica do solo está associada a maior aporte de material vegetal.

\section{Agradecimentos}

À Fundação Agrisus - Agricultura Sustentável e à Fundação de Apoio ao Desenvolvimento do Ensino, Ciência e Tecnologia do Estado de Mato Grosso do Sul, pelo apoio financeiro.

\section{Referências}

BAYER, C.; MARTIN-NETO, L.; MIELNICZUK, J.; PAVINATO, A. Armazenamento de carbono em frações lábeis da matéria orgânica de um Latossolo Vermelho sob plantio direto. Pesquisa Agropecuária Brasileira, v.39, p.677-683, 2004.

BAYER, C.; MIELNICZUK, J. Dinâmica e função da matéria orgânica. In.: SANTOS, G. de A.; CAMARGO, F.A. de O. (Ed.). Fundamentos da matéria orgânica do solo: ecossistemas tropicais e subtropicais. Porto Alegre: Genesis, 1999. p.9-26.

BAYER, C.; MIELNICZUK, J.; MARTIN-NETO, L. Efeito de sistemas de preparo e de cultura na dinâmica da matéria orgânica e na mitigação das emissões de $\mathrm{CO}_{2}$. Revista Brasileira de Ciência do Solo, v.24, p.599-607, 2000.

BAYER, C.; MIELNICZUK, J.; MARTIN-NETO, L.; ERNANI, P.R. Stocks and humification degree of organic matter fractions as affected by no-tillage on a subtropical soil. Plant and Soil, v.238, p.133-140, 2002.

BODDEY, R.M.; JANTALIA, C.P.; CONCEIÇÃO, P.C.; ZANATTA, J.A.; BAYER, C.; MIELNICZUK, J.; DIECKOW, J.; SANTOS, H.P. dos; DENARDIN, J.E.; AITA, C.; GIACOMINI, 
S.J.; ALVES, B.J.R.; URQUIAGA, S. Carbon accumulation at depth in Ferralsols under zero-till subtropical agriculture. Global Change Biology, v.16, p.784-795, 2010.

CAMBARDELLA, C.A.; ELLIOTT, E.T. Methods for physical separation and characterization of soil organic matter fractions. Geoderma, v.56, p.449-457, 1993.

CARVAlHO, P.C. de F.; ANGHINONI, I.; MORAES, A. de; SOUZA E.D. de; SULC, R.M.; LANG, C.R.; FLORES, J.P.C.; LOPES, L.M.T.; SILVA, J.L.S. da; CONTE, O.; WESP, C.L.; LEVIEN, R.; FONTANELI, R.S.; BAYER, C. Managing grazing animals to achieve nutrient cycling and soil improvement in no-till integrated systems. Nutrient Cycling in Agroecosystems, v.88, p.259-273, 2010.

CAUSARANO, H.J.; FRANZLUEBBERS, A.J.; SHAW, J.N.; REEVES, D.W.; RAPER, R.L.; WOOD, C.W. Soil organic carbon fractions and aggregation in the Southern Piedmont and coastal plain. Soil Science Society of America Journal, v.72, p.221-230, 2008.

CORAZZA, E.J.; SILVA, J.E.; RESCK, D.V.S.; GOMES, A.C. Comportamento de diferentes sistemas de manejo como fonte ou depósito de carbono em relação à vegetação de Cerrado. Revista Brasileira de Ciência do Solo, v.23, p.425-432, 1999.

DIEKOW, J. Estoque e qualidade da matéria orgânica do solo em função de sistemas de culturas e adubação nitrogenada no sistema plantio direto. 2003. 164p. Tese (Doutorado) Universidade Federal do Rio Grande do Sul, Porto Alegre.

DIEKOW, J.; MIELNICZUK, J.; KNICKER, H.; BAYER, C.; DICK, D.P.; KÖGEL-KNABNER, I. Carbon and nitrogen stocks in physical fractions of a subtropical Acrisol as influenced by long-term no-till cropping systems and $\mathrm{N}$ fertilization. Plant and Soil, v.268, p.319-328, 2005.

FRANZLUEBBERS, A.J.; STUEDEMANN, J.A. Particulate and non-particulate fractions of soil organic carbon under pastures in the Southern Piedmont USA. Environmental Pollution, v.116, p.53-62, 2002.

FUJISAKA, S.; CASTILLA, C.; ESCOBAR, G.; RODRIGUES, V.; VENEKLAAS, E.J.; THOMAS, R.; FISHER, M. The effects of forest conversion on annual crops and pastures: estimates of carbon emissions and plant species loss in a Brazilian Amazon colony. Agriculture, Ecosystems and Environment, v.69, p.17-26, 1998.

LAL, R. Soil carbon dynamics in cropland and rangeland. Environmental Pollution, v.116, p.353-362, 2002.

LEITE, L.F.C.; GALVÃO, S.R.S.; HOLANDA NETO, M.R.; ARAÚJO, F.S.; IWATA, B.F. Atributos químicos e estoques de carbono em Latossolo sob plantio direto no cerrado do Piauí. Revista Brasileira de Engenharia Agrícola e Ambiental, v.14, p.1273-1280, 2010.

LILIENFEIN, J.; WILCKE, W.; VILELA, L.; AYARZA, M.A.; LIMA, S.C.; ZECH, W. Soil fertility under native Cerrado and pasture in the Brazilian savanna. Soil Science Society of American Journal, v.67, p.1195-1205, 2003.
LOPES, M.L.T.; CARVALHO, P.C. de F.; ANGHINONI, I.; SANTOS, D.T. dos; KUSS, F.; FREITAS, F.K.; FLORES, J.P.C. Sistema de integração lavoura-pecuária: desempenho e qualidade da carcaça de novilhos superprecoces terminados em pastagem de aveia e azevém manejada sob diferentes alturas. Ciência Rural, v.38, p.1765-1773, 2008.

LOVATO, T.; MIELNICZUK, J.; BAYER, C.; VEZZANI, F. Adição de carbono e nitrogênio e sua relação com os estoques no solo e com o rendimento do milho em sistemas de manejo. Revista Brasileira de Ciência do Solo, v.28, p.175-187, 2004.

MIELNICZUK, J.; BAYER, C.; VEZZANI, F.; FERNANDES, F.F.; DEBARBA, L. Manejo de solo e culturas e sua relação com estoques de carbono e nitrogênio do solo. In: CURI, N.; MARQUES, J.J.; GUILHERME, L.R.G.; LIMAS, J.M. de; LOPES, A.S.; ALVAREZ V., V.H. (Ed.). Tópicos em ciência do solo. Viçosa: Sociedade Brasileira de Ciência do Solo, 2003. v.3, p.209-248.

NICOLOSO, R.S.; LANZANOVA, M.E.; LOVATO, T. Manejo das pastagens de inverno e potencial produtivo de sistemas de integração lavoura-pecuária no Estado do Rio Grande do Sul. Ciência Rural, v.36, p.1799-1805, 2006.

OLIVEIRA, O.C. de; OLIVEIRA, I.P. de; ALVES, B.J.R.; URQUIAGA, S.; BODDEY, R.M. Chemical and biological indicators of decline/degradation of Brachiaria pastures in the Brazilian Cerrado. Agriculture, Ecosystems and Environment, v.103, p.289-300, 2004

ROSCOE, R.; BUURMAN, P. Tillage effects on soil organic matter in density fractions of a Cerrado Oxisol. Soil and Tillage Research, v.70, p.107-119, 2003.

SALTON, J.C. Matéria orgânica e agregação do solo na rotação lavoura-pastagem em ambiente tropical. 158p. 2005. Tese (Doutorado) - Universidade Federal do Rio Grande do Sul, Porto Alegre.

SANCHEZ, P.A.; LOGAN, T.J. Myths and science about the chemistry and fertility of soil in the tropics. In: LAL, R.; SANCHEZ, P.A. (Ed.). Myths and science of soils of the tropics. Wisconsin: Soil Science Society of America, 1992. p.35-46. (SSSA. Special publication, 29).

SOUZA, E.D. de; COSTA, S.E.V.G. de A.; LIMA, C.V.S. de; ANGHINONI, I.; MEURER, E.J.; CARVALHO, P.C. de F. Carbono orgânico e fósforo microbiano em sistema de integração agricultura-pecuária submetidos a intensidades de pastejo em plantio direto. Revista Brasileira de Ciência do Solo, v.32, p.1273-1282, 2008.

TEIXEIRA, L.; LA SCALA JÚNIOR, N.; LOPES, A. Fluxo de $\mathrm{CO}_{2}$ do solo após aração e escarificação em diferentes con ções. Holos Environment, v.10, p.1-11, 2010.

VIEIRA, F.C.B. Estoques e labilidade da matéria orgânica e acidificação de um argissolo sob plantio direto afetados por sistemas de cultura e adubação nitrogenada. 2007. 124p. Tese (Doutorado) - Universidade Federal do Rio Grande do Sul, Porto Alegre. 